\title{
Sexuality and Quality of Life of Moroccan Young Woman with Breast Cancer
}

\author{
Kriouile $\mathrm{K}^{1^{*}}$, Bouchkara $\mathrm{W}^{1}$, Jayi $\mathrm{S}^{1}$, Fdili Alaoui FZ ${ }^{1}$, Chaara $\mathrm{H}^{1}$, Melhouf My $\mathrm{A}^{1}, \mathrm{O}_{\mathrm{SY}}{ }^{2}, \mathrm{~K}$. El Ghazi ${ }^{2}$ \\ ${ }^{1}$ Department of Obstetric Gynecology, HASSAN II University Hospital Center, FEZ, Morocco \\ ${ }^{2}$ Department of Epidemiology, Faculty of Medicine and Pharmacy of Fez, FEZ, Morocco
}

DOI: $10.36348 /$ sijog.2020.v03i03.004 $\quad$ | Received: 09.03.2020 | Accepted: 16.03.2020 | Published: 21.03 .2020

*Corresponding author: Kriouile $\mathrm{K}$

\section{Abstract}

This exploratory prospective study evaluated women's responses to questions that asked them to describe how their body image and sexual functioning had changed since their breast cancer diagnosis to treatment. A questionnaire concerning quality of life and various sexual problems experienced after diagnosis and treatment was anonymously completed by 200 women in the Department of Obstetric Gynecology of the University Hospital Center Hassan II of FEZ in MOROCCO. To be eligible, subjects had to be sexually active and had histology proven breast cancer. They also had to have received treatment for breast cancer. Breast cancer and its treatment may result in significant difficulties with sexual functioning and sexual life. Addressing these problems is essential to improve the quality of life of Moroccan women with breast cancer.

Keywords: Breast cancer, Sexuality, Moroccan context, Repercussions.

Copyright @ 2020: This is an open-access article distributed under the terms of the Creative Commons Attribution license which permits unrestricted use, distribution, and reproduction in any medium for non-commercial use (NonCommercial, or CC-BY-NC) provided the original author and source are credited.

\section{INTRODUCTION}

Breast cancer is the first female cancer in Morocco. The study of the impact of this type of cancer on sexuality is rarely described in the literature. The aim of this study is to assess the impact of breast cancer on quality of life and sexuality in young women $(<45$ years) to determine the factors influencing negatively on the sexual life after the diagnosis.

\section{MATERIALS AND METHODS}

This is a prospective study on the quality of life and sexual life of 200 young patients followed for breast cancer, collected over five years from January 2015 to December 2019 at the obstetric gynecology department of University Hospital Center Hassan II of FEZ (MOROCCO). The measurement of quality of life (QoL) was conducted through a questionnaire (Arabic version) associated with the proposals (questions) intended mainly to expand the theme of sexuality. Data was processed by SPSS (version 20.0).

\section{RESULTS}

The mean age was 30 years \pm 15 . The localized stage accounted for $35 \%$ of cases. At the time of the survey, $79 \%$ of patients were still on anti-tumor treatment. Changes in sexual and family life occurred after the diagnosis or after initiation of anti-tumor treatment. At diagnosis, the quality of the sexual relationship with spouse had deteriorated in $95 \%$ of cases. A Hypoactive sexual desire disorder (HSDD) was noticed in $91 \%$ of cases. Dyspareunia was reported by $79 \%$ of subjects. The sexual relationship was fulfilling in $85 \%$ of cases, with an influence on the emotional balance in $95 \%$ of cases. Several psychosocial and sociodemographic factors are implicated in the deterioration of sexual life. This is among other lower socioeconomic level (52\%), acceptance of body image after mastectomy (32\% of cases), and chemotherapy-induced menopause with vaginal dryness signs in $53 \%$ of cases. Only one patient had a child after the end of antitumor treatment. The main reason for their decision not to have children was the age at diagnosis or beliefs. The relationship with the spouse had deteriorated in $55 \%$ of cases with $17 \%$ 
separation and divorce $10 \%$ at diagnosis or during treatment.

\section{DISCUSSION}

Over the past thirteen years a great deal of effort has been expended to enhance the possibility of early diagnosis and improve the treatment of breast cancer, and problems related to the disease have been extensively investigated. It is now clear that the psychological problems most frequently observed in these patients are depression, body image-associated hypochondria and sexual dysfunction [1,2]. Our study, aimed to assess in treated breast cancer Moroccan women the subjective disturbance of sexual dysfunction that often is not expressed and that contributes to further lowering the quality of life. The interviews revealed a number of notable findings regarding sexuality after breast cancer in the Moroccan context. The sexual impact of having cancer and its treatments have long been a taboo topic in hospital settings in Morocco. The sexual aspects of having cancer have long been neglected in medical practice. There are a number of barriers to the discussion of sexual issues by patients and professionals. Difficulties related to sexuality and sexual functioning was common and occurred since their breast cancer diagnosis to treatment. The majority of informants revealed that they had experienced sexual dysfunction and physical discomfort caused by the breast cancer treatment. Patients reported worse sexual functioning, characterized by greater lack of sexual interest, inability to relax and enjoy sex, difficulty becoming aroused, and difficulty reaching the orgasm. Regarding personal and sexual relationships, $85 \%$ had an important personal relationship (partner) and 95\% were effectively sexually active; following diagnosis and various treatments of breast cancer. $55 \%$ of the subjects had ceased sexual relations and $10 \%$ divorced. However, we were not able to compare the data from this study regarding sexual problems with those of other groups (normal population or samples of subjects with chronic disease) since to date no epidemiological studies of sexual behaviour and distribution of sexual dysfunctions in the normal population have been performed in Morocco. Nor was it possible to make a comparison between the data obtained from this study and that which had emerged from research carried out in other countries because of cultural, social, economic and moral variables which enormously condition sexual behaviour. Nevertheless, patients of this study had lower scores in the all body image subscales. This finding is comparable with other studies [3-6]. Howighorst-Knapstein and al also found that, mastectomy resulted in lower sexual desire and changes in body image [7]. Bakwell \& Volker also showed that all types of treatment for breast cancer had a significant impact on body image and menopausal status and finally results in sexual problems [8]. In Summary, breast cancer affects many aspects of a Moroccan woman's sexuality, including changes in physical functioning and in perception of femaleness but this study has some limitations. The first limitation of this study is the sample structure which was limited to breast cancer women and didn't study healthy women with these problems. The data obtained from this study must be interpreted with caution since they reflect the limited methodology with which the research was conducted. The sample seems to be small and not representative enough to allow us to extrapolate the data for a larger population. The second limitation of this study is the missing role of husband/ partner in the study which can have a very important role in patient life. However, this study show that the onset of sexual dysfunctions in concomitance with and after treatment of breast cancer is frequent and that such dysfunctions noticeably compromise the quality of sex life and does sufficiently address a problem that needs to be investigated in greater depth. Although it is not possible to make distinctions, it appears that the most numerous dysfunctions are those which originate easily from compromises in physical state (dyspareunia and lubrication difficulties) while the fewest dysfunctions were of a psychological nature (the absence or reduction of sexual desire, difficulty to reach orgasm and brevity of intercourse). These disturbances, even though they noticeably reduce the quality of sex life, do not compromise it completely, as the majority of subjects remain sexually active [9].

\section{CONCLUSION}

The results presented emphasize the high prevalence of sexual dysfunction in patients with breast cancer. Various factors appear associated with a negative impact on sexuality. Sexual function disorders should be considered in a comprehensive, just like the other organic, psychological or social problems.

\section{REFERENCES}

1. Morris, T., Greer, S., Pettingale, K. W., \& Watson, M. (1981). Patterns of expression of anger and their psychological correlates in women with breast cancer. Journal of psychosomatic research, 25(2), 111-117.

2. Schover, L. R. (1991). The impact of breast cancer on sexuality, body image, and intimate relationships. CA: a cancer journal for clinicians, 41(2), 112-120.

3. Fobair, P., Stewart, S. L., Chang, S., D'Onofrio, C., Banks, P. J., \& Bloom, J. R. (2006). Body image and sexual problems in young women with breast cancer. Psycho- Oncology: Journal of the Psychological, Social and Behavioral Dimensions of Cancer, 15(7), 579-594.

4. Shoma, A. M., Mohamed, M. H., Nouman, N., Amin, M., Ibrahim, I. M., Tobar, S. S., ... \& William, S. G. (2009). Body image disturbance and surgical decision making in egyptian post menopausal breast cancer patients. World Journal of Surgical Oncology, 7(1), 66. 
5. Garrusi, B., \& Faezee, H. (2008). How do Iranian women with breast cancer conceptualize sex and body image?. Sexuality and Disability, 26(3), 159165.

6. Helms, R. L., O'Hea, E. L., \& Corso, M. (2008), Body image issues in women with breast cancer. Psychology, Health and medicine, 13(3), 313-325.

7. Hawighorst-Knapstein, S., Fusshoeller, C., Franz, C., Trautmann, K., Schmidt, M., Pilch, H., ... \& Vaupel, P. (2004). The impact of treatment for genital cancer on quality of life and body image- results of a prospective longitudinal 10-year study. Gynecologic oncology, 94(2), 398-403.

8. Bakewell, R. T., \& Volker, D. L. (2005). Sexual dysfunction related to the treatment of young women with breast cancer. Clinical journal of oncology nursing, 9(6), 697.

9. Sbitti, Y., Kadiri, H., Essaidi, I., Fadoukhair, Z., Kharmoun, S., Slimani, K., ... \& Errihani, H. (2011). Breast cancer treatment and sexual dysfunction: Moroccan women's perception. BMC women's health, 11(1), 29. 